\title{
Development of Safe Mechanism for Surgical Robots Using Equilibrium Point Control Method
}

\author{
Shinsuk Park, Hokjin Lim, Byeong-sang Kim, and Jae-bok Song \\ Dept. of Mechanical Engineering, Korea University, \\ Anam-dong, Sungbuk-gu, Seoul, Korea \\ \{drsspark, jini0117, lovidia, jbsong\}@korea.ac.kr
}

\begin{abstract}
This paper introduces a novel mechanism for surgical robotic systems to generate human arm-like compliant motion. The mechanism is based on the idea of the equilibrium point control hypothesis which claims that multijoint limb movements are achieved by shifting the limbs' equilibrium positions defined by neuromuscular activity. The equilibrium point control can be implemented on a robot manipulator by installing two actuators at each joint of the manipulator, one to control the joint position, and the other to control the joint stiffness. This double-actuator mechanism allows us to arbitrarily manipulate the stiffness (or impedance) of a robotic manipulator as well as its position. Also, the force at the end-effector can be estimated based on joint stiffness and joint angle changes without using force transducers. A two-link manipulator and a three-link manipulator with the double-actuator units have been developed, and experiments and simulation results show the potential of the proposed approach. By creating the human arm-like behavior, this mechanism can improve the performance of robot manipulators to execute stable and safe movement in surgical environments by using a simple control scheme.
\end{abstract}

\section{Introduction}

Over the past years, robotic technology has been rapidly gaining acceptance in the operation room. Robots are known to enhance surgery by improving precision, repeatability, stability, and dexterity [1]. Surgical robots are now regularly used in minimally invasive surgery (MIS). Robots for MIS procedures are required to safely perform contact tasks with the patient body in a small workspace.

A robot that contacts the surrounding environment and works within kinematic constraints requires force control, and must be capable of accurately modulating and controlling its actuator torques and forces in addition to knowing where it is in its workspace. Such robots, however, have mainly been limited to laboratory research so far.

Unlike robot manipulators, humans are capable of autonomously performing a wide range of tasks in unpredictable environments. They particularly excel at contact tasks, such as opening a door, turning a knob or lever, and turning a key in a lock. They use a combination of intrinsic compliance, forces and torque sensation, and tactile contact clues from the environments. The human arm muscles are compliant 
actuators that are arranged antagonistically about joints to independently control the multi-dimensional position and compliance at the limb endpoint. The main characteristic of the human arm is that it introduces relatively high, controllable compliance at the mechanical level: rather than achieving compliance by methods based on controller synthesis, the human arm has redundant actuators that provide intrinsic compliance and thus safety [2]. Several studies employed redundant actuators to modulate stiffness of robot manipulators [3],[4].

The purpose of this paper is to present a novel mechanism for surgical robotic systems by adopting the idea of the equilibrium point control hypothesis, one of many theories in human motion control. This study proposes to convert the human motor control scheme into a novel robot control scheme using the double-actuator mechanism, which employs two actuators for each joint: one for position control and the other for stiffness control. A two-link manipulator and a three-link manipulator with the double-actuator units have been developed, and experiments and simulation were conducted to show the validity of this mechanism in surgical environments.

\section{Double-Actuator Mechanism}

While a great number of studies have attempted to reveal the basic properties of the human neuromuscular control system, understanding the actual control strategies adopted by the CNS is a fundamental problem of neurophysiology yet to be explored [5],[6]. A simple approach to this problem suggests that the CNS utilizes the effective dynamic and mechanical properties of the muscles and neural feedback circuits to circumvent the computational complexities. For a single joint, the combined action of a group of muscles spanning the joint, both agonists and antagonists, determines an equilibrium posture for the joint. Central command generates a series of equilibrium points for a limb, and the "spring-like" properties of the neuromuscular system will drive the motion along a trajectory that follows these intermediate equilibrium postures. This equilibrium point control hypothesis applies to the control of both static posture and dynamic movement [7]. In the framework of equilibrium point control, the resultant joint torques are determined only by deviation of the actual trajectory from the equilibrium point trajectory and joint impedance.

It should be noted that equilibrium point control applies the same strategy to tasks requiring interaction with the environment, unrestrained motions and the transition between the two. Control of contact force can be achieved through the use of an equilibrium point, as well as the controlled joint stiffness: simply moving the equilibrium point to a point within a contact object will cause the limb to exert a force on that object.

One way to implement the control scheme would be placing the positioning actuator and the stiffness modulator in series on one joint axis. In this study we employed an electrical motor with a higher gear ratio as the positioning actuator, and an electrical motor with a lower gear ratio as the stiffness modulator. Fig. 1 (a) illustrates the two actuators placed on one joint axis. With the mechanism, we can control the equilibrium point and joint stiffness at the same time: one actuator commands the equilibrium point (positioning actuator), the other joint stiffness (stiffness modulator). The positioning actuator provides the equilibrium point (angle), and the stiffness modulator controls the 
restoring torque around the point. The positioning actuator is fixed to the base link, and the moving link is fixed to the output axis of the stiffness modulator. Fig. 1 (b) shows an equivalent mechanism to the one in Fig. 1 (a). Two actuators are connected in parallel by using a gear train: one gear is fixed to the output axis of the positioning actuator, the other to the frame of the stiffness modulator.

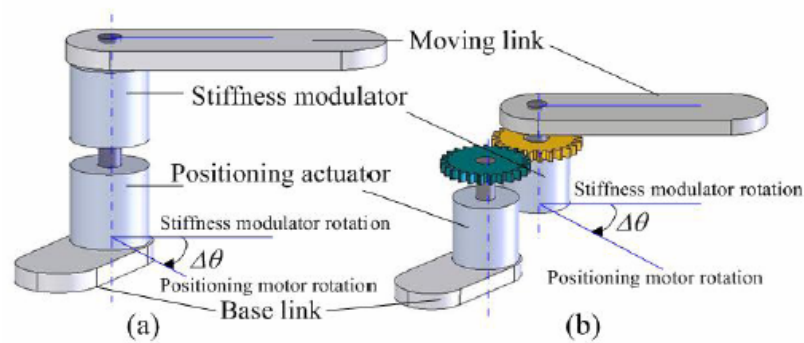

Fig. 1. Conceptual figures of two equivalent double actuator mechanisms (a) two actuators connected in series, and (b) two actuators connected in parallel using a gear train

Fig. 2 illustrates the double-actuator mechanism we have developed based on the idea of Fig. 1 (b). For the positioning actuator and stiffness modulator of the manipulator, BLDC motors were used, where the stiffness modulator had a lower gear ratio for back-drivability when attempting compliant motion control using the proposed control scheme.
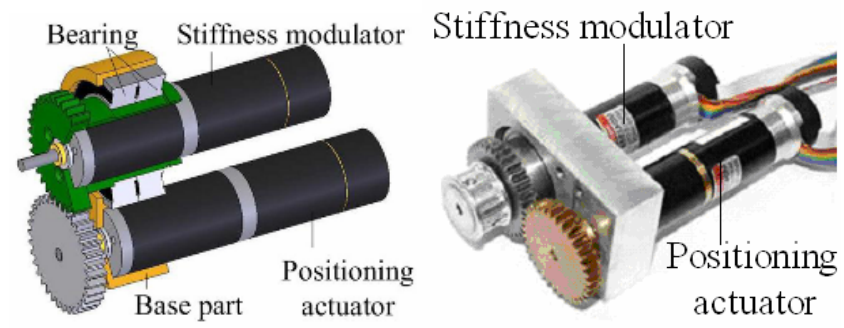

Fig. 2. Double-actuator mechanism with two parallel actuators

\section{Manipulators with Double-Actuator Units}

When the manipulator equipped with the double-actuator mechanism is in contact with an object, the contact force can be controlled either by moving the equilibrium point further into the contacting object or by modulating the stiffness at the endpoint of the manipulator. For a desired endpoint stiffness matrix $\mathbf{K}_{x}$ in the Cartesian coordinate system, the joint stiffness matrix $\mathbf{K}_{J}$ of the stiffness modulator can be calculated using the manipulator Jacobian $\mathbf{J}_{M}$ as follows: 


$$
\mathbf{K}_{J}=\mathbf{J}_{M}{ }^{T} \mathbf{K}_{x} \mathbf{J}_{M}
$$

The contact force $\mathbf{F}$ at the endpoint can be simply estimated by using the following relationship:

$$
\mathbf{F}=\mathbf{J}_{M}{ }^{-T} \boldsymbol{\tau}=\mathbf{J}_{M}{ }^{-T}\left(\mathbf{K}_{J} \Delta \boldsymbol{\theta}\right)=\mathbf{J}_{M}{ }^{-T}\left(\mathbf{J}_{M}{ }^{T} \mathbf{K}_{x} \mathbf{J}_{M}\right) \Delta \boldsymbol{\theta}=\mathbf{K}_{x} \mathbf{J}_{M} \Delta \boldsymbol{\theta}
$$

where $\tau$ is the torque exerted by the stiffness modulator and $\Delta \theta$ is the difference in joint angles between the equilibrium posture and actual posture. Since the stiffness modulator is capable of active stiffness modulation based on angle measurements, the joint stiffness matrix $\mathbf{K}_{J}$ realized by the stiffness modulator contains off-diagonal terms, unlike passive compliant elements discussed in introduction.

\subsection{Manipulation of Stiffness Ellipse Using Two-Link Manipulator}

Fig. 3 shows a two-link manipulator equipped with the double actuator mechanism described in the previous section. The double-actuator units that drive the first and the second link are attached to the base and the first link, respectively. The driving torque of the double-actuator units were transferred to the links by timing pulleys and timing belts. Encoders were equipped to the output axis of each actuator to obtain position feedback. With the two-link manipulator, stiffness matrices at the endpoint can be manipulated arbitrarily in 2D space. Simply by manipulating stiffness ellipse, we can easily control the magnitude and direction of the contact force between the robot and the environment. Also, the double-actuator mechanism allows us to estimate contact force only by the angle measurements at the joints, without using a force transducer at the endpoint.

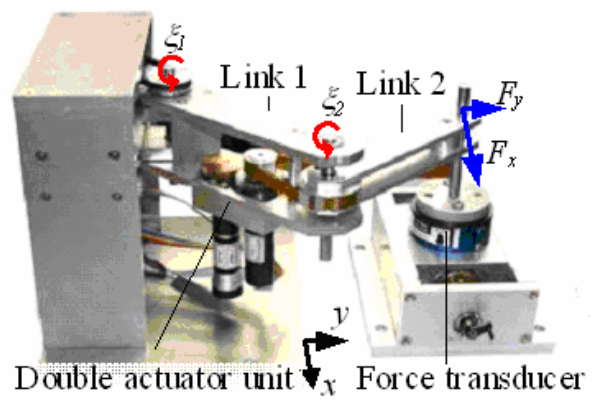

Fig. 3. Two-link manipulator with double actuator mechanism

Experiments. With a given endpoint stiffness matrix in Cartesian coordinate system, force-displacement relation at the endpoint was measured by using a force transducer (JR3 J409). With the given stiffness matrices, the equilibrium point of the endpoint was moved in six different directions so that force-displacement relation can be measured in the directions. In this experiment, three stiffness matrices were given for three different configurations of the manipulator. Fig. 4 compares the target stiffness 
ellipses and the estimated stiffness ellipses. As can be seen in the figure, the actual stiffness is nearly identical to the target stiffness in all the configurations.

Fig. 5 compares the measured and estimated contact forces while the endpoint is in contact with an object. The estimated force was based on encoder data from the two double-actuator units in the two-link manipulator. The figure demonstrates that the estimated force is very close to the measured force.
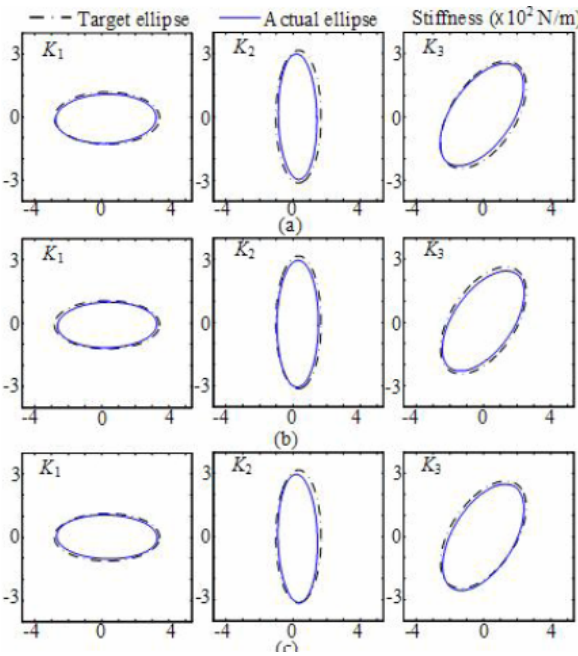

Fig. 4. Comparison of target and actual stiffness ellipse

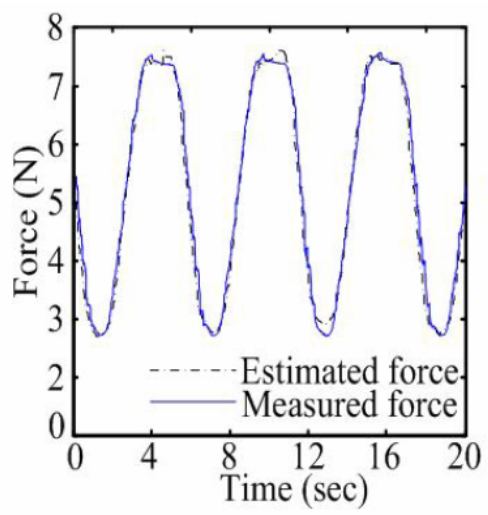

Fig. 5. Comparison of measured and estimated force

\subsection{Adaptive Control of Remote Compliance Center Using Three-Link Manipulator}

In MIS, surgical procedures are carried out using long instruments inserted into the patient's body through small incisions with the surgeon manipulating the tool handles from outside the patient's body. The remote center of motion (RCM) that constrains the motion of the instrument through a known fulcrum point has been used in several laparoscopic robots, including commercial systems such as the da Vinci (Intuitive surgical) [8]. With the RCM method, however, a small error in the fulcrum position can cause a large contact force between the patient body and the instrument.

Remote compliance center (RCC) devices have long been used to facilitate insertion for assembly operations in manufacturing [9]. By decoupling lateral and angular motion around the compliance center, a RCC device can ease the contact force during insertion of a part into a hole. When a project compliance center is near the insertion point, the part translates into the hole when it strikes the outside without rotating. This translation without rotation prevents the jamming and galling, which can generate excessive contact force. Since conventional RCC devices employ passive mechanical compliance, the compliance center is fixed at the tip of the part to be inserted and moves further into the hole after the part is inserted. 


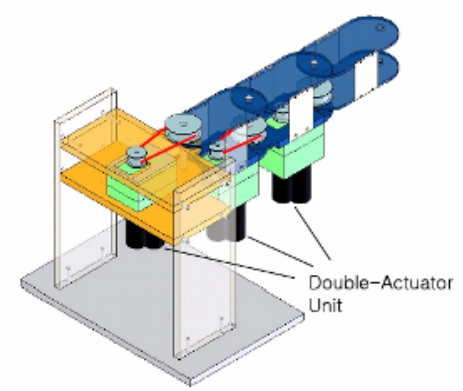

Fig. 6. Three-link manipulator with double actuator mechanism

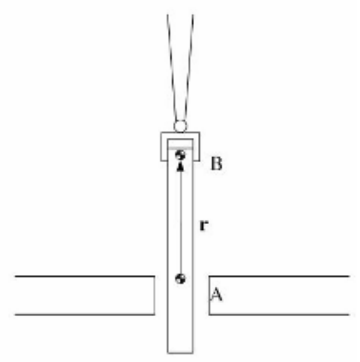

Fig. 7. Insertion of an instrument into a port

By implementing three double-actuator units on a three-link manipulator, the position of the compliance center can be controlled in two-dimensional space so that the compliance center is maintained near the insertion point during the whole insertion operation (Fig. 6). This adaptive control of RCC, when applied to MIS, can be advantageous, since it prevents excessive force at the port site where the surgical instrument or endoscope is inserted. Fig. 7 illustrates the insertion of a surgical instrument into a port. The position of the endpoint of the robot manipulator (point B) with respect to the insertion point (point A) is represented by vector $\mathbf{r}\left(\mathbf{r}=\left(r_{x}, r_{y}\right)^{T}\right)$.

Using the double-actuator mechanism, the compliance center can be placed at the insertion point (point $\mathrm{A}$ ), and the compliance matrix described at point $\mathrm{A}$ is given as:

$$
\mathbf{C}_{A}=\left[\begin{array}{ccc}
C_{x x} & C_{x y} & 0 \\
C_{y x} & C_{y y} & 0 \\
0 & 0 & C_{\theta \theta}
\end{array}\right]=\left[\begin{array}{cc}
\mathbf{C}_{r} & \mathbf{0} \\
\mathbf{0} & C_{\theta}
\end{array}\right]
$$

The compliance matrix is transformed by Jacobian matrix $\mathbf{J}$ between point $\mathrm{A}$ and point B to yield the compliance matrix at the endpoint of the manipulator (point B).

$$
\mathbf{C}_{B}=\mathbf{J C}_{A} \mathbf{J}^{T}=\left[\begin{array}{cc}
\mathbf{C}_{r}+\mathbf{r}^{*} \mathbf{C}_{\theta} \mathbf{r}^{* T} & \mathbf{r}^{*} \mathbf{C}_{\theta} \\
\mathbf{C}_{\theta} \mathbf{r}^{* T} & \mathbf{C}_{\theta}
\end{array}\right]
$$

where $\mathbf{r}^{*}=\left(-r_{y}, r_{x}\right)^{T}$.

With the predetermined compliance matrix $\mathbf{C}_{A}$ at the insertion point and the vector $\mathbf{r}$ that can be estimated from forward kinematics of the manipulator, we can calculate the compliance matrix $\mathbf{C}_{B}$. The joint stiffness $\mathbf{K}_{\theta}$ for the manipulator can be determined using the manipulator Jacobian $\mathbf{J}_{M}$ :

$$
\mathbf{K}_{\theta}=\mathbf{J}_{M}{ }^{T} \mathbf{C}_{B}{ }^{-1} \mathbf{J}_{M}
$$

Simulations. Using a commercial dynamic simulation software (MSC Visual Nastran), we performed simulations to compare the performance of the adaptive RCC 
method with conventional RCC devices. Fig. 8 compares the contact impulse during insertion of a long instrument into a port. While the first peak vales are nearly the same, the second peak impulse with the adaptive RCC control is lower than that of conventional RCC. Fig. 9 compares the contact impulse while the instrument is moving laterally while inserted halfway into the hole. Compared to conventional RCC, we can significantly reduce the contact force with the adaptive RCC control.

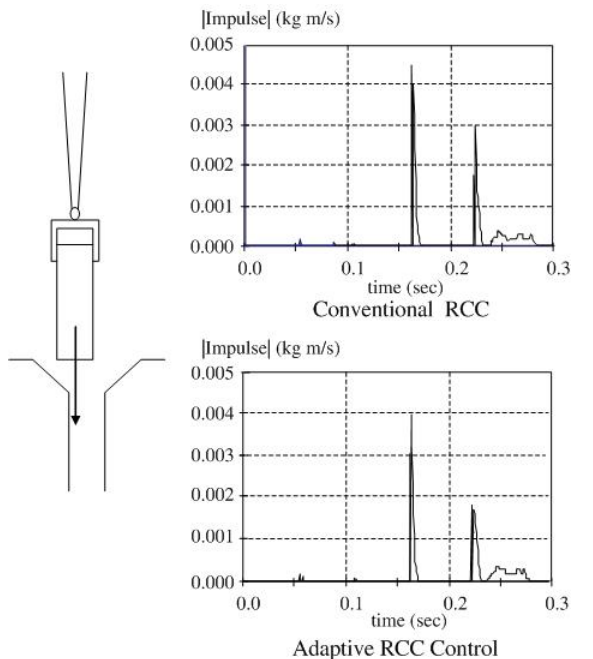

Fig. 8. Comparison of contact impulse during insertion

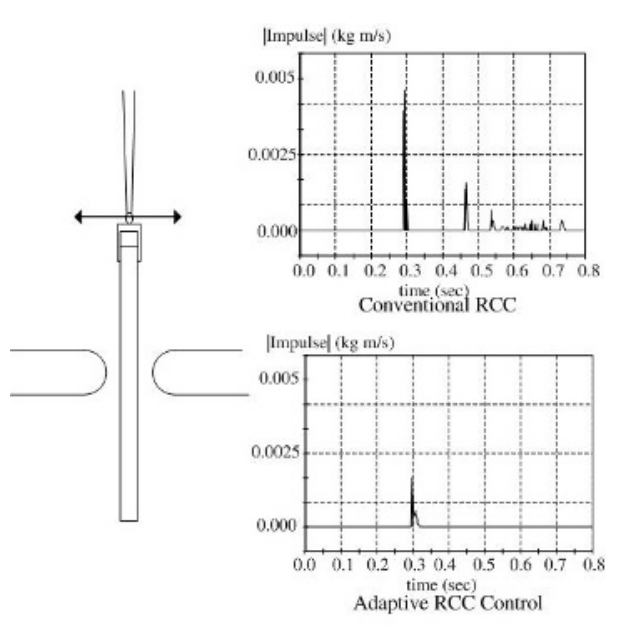

Fig. 9. Comparison of contact impulse during lateral motion

\section{Conclusions}

This work proposes a novel mechanism for robot manipulators based on the equilibrium point control scheme. Experimental results demonstrated that the mechanism could successfully control position and compliance of the robot under the equilibrium point control scheme.

There are several benefits of the proposed control scheme. One of the benefits would be the simplicity of control. Equilibrium point control applies the identical strategy from motion in free space to contact task. In cases when in contact, the contact force can be applied by simply moving the equilibrium point within the contact object. Contact force can also be tailored by tuning the compliance of the second motor. Secondly, equilibrium point control turns the force control problem into a position control problem. In this control scheme, output torque is proportional to the difference in the angles, where the angles are much easier to sense and control than the torques. In many force control schemes, force/torque sensors are equipped near the endeffector to detect the contact force at the end-effector. It is difficult, however, to detect contact on the parts far from the end-effector. The developed mechanism estimates the contact force based on the angle measurement at the joints. Thus, the contact on the part far from the end-effector can also be detected and estimated. 
A conceivable application of the developed mechanism would be teleoperation in surgical environments, where robotic systems are required to regenerate the movement of the surgeon while regulating the contact force under a safe level. There are several issues to be considered, including the size of mechanism and the extension of DOFs. These issues will be studied in our future study.

\section{Acknowledgment}

This work was supported by grant No. (R01-2003-000-10336-0) from the Basic Research Program of the Korea Science \& Engineering Foundation.

\section{References}

[1] Howe, R., Matsuoka, Y.: Robotics for Surgery. Ann. Rev. Biomed. Eng. (2000) 1: 211-240.

[2] Tonietti, G., Schiavi, R., Bicchi, A.: Design and Control of a Variable Stiffness Actuator for Safe and Fast Physical Human/Robot Interaction. Proc. of IEEE Int'l Conf. on Robotics and Automation (2005) 528-533.

[3] Yi, B., Freeman, R., Tesar, D.: Open-loop Stiffness Control of Overconstrained Mechanisms/Robotic Linkage Systems. Proc. of IEEE Int'l Conf. on Robotics and Automation (1989) 1340-1345.

[4] Yi, B., Freeman, R., Tesar, D.: Geometric Characteristics of Antagonistic Stiffness in Redundantly Actuated Mechanisms. Proc. of IEEE Int'l Conf. on Robotics and Automation (1993) 654-661.

[5] Bernstein, N.: The Coordination and Regulation of Movements. Pergamon Press (1967).

[6] Arbib, M.: The Metaphorical Brain: An Introduction to Artificial Intelligence and Brain Theory. Interscience (1972).

[7] Bizzi, E., Hogan, N., Mussa-Ivaldi, F., Giszter, S.: Does the Nervous System Use Equilibrium-Point Control to Guide Single and Multiple Joint Movements. Behavioral and Brain Sciences, Vol. 15 (1992) 603-613.

[8] Taylor, R., Funda, J., Eldridge, B., Gruben, K., LaRose, D., Gomory, S. Talamini, M., Kavoussi, L., Anderson, J.: A Telerobotic Assistant for Laparoscopic Surgery. IEEE EMBS Magazine Special Issue on Robotics in Surgery (1995) 279-291.

[9] Whitney, D.: Part Mating in Assembly. Handbook of Industrial Robotics (1985) 1084-1116. 